\title{
Stage IB Gastric Gastrointestinal Stromal Tumor
}

National Cancer Institute

\section{Source}

National Cancer Institute. Stage IB Gastric Gastrointestinal Stromal Tumor. NCI

Thesaurus. Code C87826.

Stage IB includes: T3, NO, M0, Mitotic rate: Low. T3: Tumor more than $5 \mathrm{~cm}$, but not more than $10 \mathrm{~cm}$, in greatest dimension. N0: No regional lymph node metastasis. MO: No distant metastasis. (AJCC 7th ed.) 